\title{
Abnormalities in the 24-hour Rhythm of Skin Temperature in Cirrhosis: Sleep-Wake and General Clinical Implications
}

\author{
Maria Garrido1, Desy Saccardo1, Michele De Rui1, Elia Vettore1, Alberto Verardo1, Paolo \\ Carraro2, Nicola Di Vitofrancesco3, Ali R. Mani4, Paolo Angeli1, Massimo Bolognesi1, Sara \\ Montagnese 1
}

1Department of Medicine, University of Padova, Padova, Italy 2Laboratorio Analisi, Azienda ULSS 12 Veneziana, Mestre, Italy

3Department of Laboratory Medicine, University-Hospital, Padova, Italy

${ }_{4}$ Division of Medicine, University College London, London, UK

\section{Correspondence to:}

Sara Montagnese

Dipartimento di Medicina

Via Giustiniani, 2

35128 Padova, Italia

Tel +390498218675

Fax +390497960903

sara.montagnese@unipd.it

Abbreviations: C-Reactive Protein, CRP; Distal-Proximal Skin temperature Gradient, DPG; Epworth Sleepiness Scale, ESS; Horne-Östberg, HÖ; Interleukin-6, IL-6; Intra-assay Coefficient of Variation, CV; Model for End-stage Liver Disease, MELD; Multiscale Entropy, MSE;

Pittsburgh Sleep Quality Index, PSQI; Receiver Operating Characteristic, ROC; Tumor Necrosis Factor-alpha, TNF- $\alpha$.

Funding: The work was partly funded by a grant from the Italian Ministry of Health to S.M. (GR 2009-1604309); M.G. holds a post-doctoral fellowship from Gobierno de Extremadura [jointly financed by the European Regional Development Fund; ref. PO14013].

Conflict of Interests: None 


\begin{abstract}
Background \& Aims: Sleep preparation/onset are associated with peripheral vasodilatation and a decrease in body temperature. The hyperdynamic syndrome exhibited by patients with cirrhosis may impinge on sleep preparation, thus contributing to their difficulties falling asleep. The aim of this study was the assessment of skin temperature, in relation to sleep-wake patterns, in patients with cirrhosis.
\end{abstract}

Methods: Fifty three subjects were initially recruited, and 46 completed the study. Of the final 46, twelve were outpatients with cirrhosis, 13 inpatients with cirrhosis, 11 inpatients without cirrhosis and 10 healthy volunteers. All underwent baseline sleep-wake evaluation and blood sampling for inflammatory markers and morning melatonin levels. Proximal/distal skin temperature and their gradient (DPG) were recorded for 24 hours by a wireless device. Over this period subjects kept a sleep-wake diary.

Results: Inpatients with cirrhosis slept significantly less well than the other groups. Inpatients and outpatients with cirrhosis had higher proximal temperature and blunted rhythmicity compared to the other groups. Inpatients with/without cirrhosis had higher distal temperature values and blunted rhythmicity compared to the other groups. Inpatients and outpatients with cirrhosis had significantly lower DPG values compared to the other groups, and DPG reached near-zero values several hours later. Significant correlations were observed between temperature and sleep-wake variables and inflammatory markers.

Conclusions: Alterations of distal/proximal skin temperature, their gradient and their time-course were observed in patients with cirrhosis, which may contribute to their sleep disturbances.

Keywords: circadian rhythm, sleep latency, inflammatory marker, hepatic failure.

\title{
Key points
}

$\checkmark$ In the healthy population, the gradient between distal and proximal skin temperature (DPG) is an excellent predictor of sleep latency, with a near-zero DPG indicating that sleep is imminent.

Patients with cirrhosis exhibited significantly impaired thermoregulation, both in terms of absolute values and the time-course of DPG.

Significant correlations were observed between skin temperature and sleep-wake indices, suggesting that abnormal thermoregulation may contribute to the sleep-wake disturbances exhibited by these patients.

$\square$ These findings are likely to have clinical implications for both the definition of fever and the management of insomnia in patients with cirrhosis. 


\section{Introduction}

Sleep is regulated by the interaction between a homeostatic and a circadian process. 1 The homeostatic process determines sleep propensity in relation to sleep-wake history, so that the need to sleep increases with the duration of wakefulness.2 The circadian process, which is marked by variations in the plasma levels of the hormone melatonin, regulates sleep propensity in relation to dark/light cues. Sleep preparation is accompanied by changes in vascular tone and peripheral temperature, which are key to the timing and the speed of sleep onset.3,4 Accordingly, an index of peripheral heat loss [i.e. the distal-proximal skin temperature gradient (DPG)] is an excellent predictor of sleep latency, or the length of time between attempting to sleep and actual sleep onset. When the DPG reaches near-zero values, sleep is imminent.3,5

A significant proportion of patients with cirrhosis exhibit sleep-wake disturbances, mainly in the form of difficulties falling asleep, awakenings, alterations in sleep timing, and excessive daytime sleepiness.6,7 Despite multiple theories, the pathophysiology of such disturbances remains largely unknown. Hepatic encephalopathy is thought to play a role, especially in daytime sleepiness. 8 Alterations in both circadian9 and homeostatic sleep-wake regulation have been documented,10-11 and an abnormal interaction between the two systems is thus likely.12

There is a yet unexplored possibility that the splanchnic vasodilatation/hyperdynamic circulatory syndrome which complicates cirrhosis may impinge on temperature regulation, and thus compromise the fine temperature changes that accompany sleep preparation and sleep onset. Thus the aim of this study was to determine the 24-hour distal and proximal temperature curves and the DPG of both in- and out-patients with cirrhosis, with reference to their sleep-wake patterns and by comparison with healthy and disease controls.

\section{Methods \\ Subjects}

A total of 53 subjects were initially enrolled: 16 inpatients with cirrhosis, 16 outpatients with cirrhosis, 11 inpatients without cirrhosis and 10 healthy volunteers. Patients were excluded if they were $<18$ years, active smokers, could not/were unwilling to comply with the study procedures, had a fever or infection, had cancer, a history of neurological/psychiatric comorbidity, were taking beta-blockers, drugs affecting the vascular tone or psychoactive drugs (for example hypnotics, anxiolytics and neuroleptics). Patients and healthy volunteers were excluded if they had misused alcohol or undertaken shift work/intercontinental travel in the preceding six months. The aetiology of cirrhosis was established on clinical, laboratory, radiological, and histological variables. Its severity was determined using Pugh's modification of the Child's grading system 13 and the model for end-stage liver disease (MELD) score.14

\section{Experimental design}

One experimental session was conducted over 28 hours, from 08:00 am to 12:00 midday of the following day (Figure 1). All participants completed a baseline sleep-wake assessment and then a detailed sleep-wake diary covering the experimental study session. Temperature recordings were carried out over 24 hours (12:00 midday to 12:00 midday of the following day) by use of temperature loggers. Blood samples were collected at the beginning of the experimental session, at 8:00 am.

\section{Baseline sleep-wake profile}

The Pittsburgh Sleep Quality Index (PSQI) questionnaire is utilized to assess subjective sleep quality over the preceding month. The higher the PSQI global score (range 0-21), the worst the sleep quality. A score $\geq 5$ identifies poor sleepers.15,16

The Epworth Sleepiness Scale (ESS) is used to evaluate the daytime sleepiness. Individuals rate their likelihood of 'dozing off' in eight different daytime situations. The higher the ESS score (range 0-24), the sleepier the subject. A score of $\geq 11$ is considered abnormal.17 The HorneÖstberg (HÖ) questionnaire evaluates diurnal preference and qualifies subjects as definitely 
morning, moderately morning, intermediate, moderately evening and definitely evening based on 19 self-administered questions. 18

\section{Sleep-wake diary}

Subjects were instructed to fill in the wake diary (daily activities, meal times and any period/reason for removal of the sensors) before going to bed and the sleep diary (bed time, sleep onset time, try to sleep time, wake-up time, get-up time, number of any night awakening/daytime naps) the following morning. Standard indices were obtained, to include sleep onset latency (or time required to fall asleep), number of awakenings, total time spent in bed, sleep efficiency (i.e., time asleep/time spent in bed, as a percentage), and sleep quality on a scale of 1 to 10.19 For subjects who reported long and difficult-to-estimate sleep latencies, a fixed, arbitrary maximum of $120 \mathrm{~min}$ was utilized (3 inpatients with cirrhosis and 1 inpatient without cirrhosis). Similarly, for subjects who reported several awakenings during the night, a fixed, arbitrary maximum of 10 awakenings was utilized ( 2 inpatients with cirrhosis and 3 inpatients without cirrhosis).

\section{Distal and proximal temperature}

Distal and proximal temperatures were recorded for 24 hours by use of wireless temperature sensors (iButtons, model no. DS1922L-F5, Maxim Integrated, San Jose, CA, USA). 20-21 Sampling rate was set at $3 \mathrm{~min}$ (resolution $0.0625^{\circ} \mathrm{C}$; approximately 500 temperature values per day). Nine sensors were placed on the skin (left/right mid-thighs on the rectus femoris, left/right infraclavicular area, abdomen, thenar area of the left/right hand, and mid metatarsal area of the plantar site of the left/right foot) using medical tape. Data from the sensors were transferred by an adapter to a computer, using the iButton Viewer software (Dallas Semiconductor, Maxim Integrated Products, Sunnyvale, CA). An artifact rejection procedure was applied to exclude extremes.20 Then proximal and distal skin temperature were calculated as follows: 20,22 $T_{\text {prox }}=0.383 \times$ average $($ mid-thighs $)+0.293 \times$ average $($ infraclavicular sites $)+0.324 \times$ abdomen $T_{\text {dist }}=[$ average $($ hands $)+$ average $($ feet $)] / 2$

The above were then used to calculate DPG $\left(T_{\text {dist }}-T_{p r o x}\right)$.

\section{Temperature variability analysis}

Methods derived from nonlinear dynamics are useful in analyzing the complexity of physiological signals such as heart rate, respiratory rate and temperature.23 Body temperature exhibits complex fluctuations at different time-scales and analyzing pattern of these variations provides information about the dynamics of the thermoregulatory system.23-24 Proximal temperature data were used for calculation of sample entropy and multiscale entropy in this study. Sample entropy

Sample entropy measures the negative logarithmic likelihood of the repetition of patterns in a time series. In other words, sample entropy calculates the probability that subseries within a temperature series that match within tolerance $r$ for $m$ points will also match for $m+1$ points, taking into account the fact that subseries might overlap. A lower value of sample entropy reflects a higher degree of regularity. In the present study, the parameter $m$ was fixed to 2 , and tolerance level $\mathrm{r}$ was 0.2 .

Multiscale entropy (MSE)

Multiscale entropy (MSE) measures the irregularity of a time series as a function of time scale. 25 The temperature-series of different time scales were reconstructed by averaging non-overlapping consecutive $n$ temperature data to form the new time-series (scaling factors from 1 to 10 were used in this study). Entropy in each new time series was quantified by calculating sample entropy as described before. 25

\section{Laboratory variables}

Venous blood samples $(5 \mathrm{ml})$ were obtained at 08:00 and stored in plastic tubes containing clotting activator (BD-Vacutainer, ref. 368815). Samples were then centrifuged at $1500 \times \mathrm{g}$ for $10 \mathrm{~min}$ at $4{ }^{\circ} \mathrm{C}$. The resulting serum was divided into $1 \mathrm{ml}$ aliquots and stored at $-80{ }^{\circ} \mathrm{C}$ until they were analyzed.

\section{Cytokines determination}


Interleukin-6 (IL-6) and tumor necrosis factor-alpha (TNF- $\alpha$ ) were measured using a solid-phase immunological method with a monoclonal immobilizing murine antibody and a polyclonal enzyme-labeled (bovine alkaline phosphatase) antibody. The system was coupled in a chemoluminescent sequential immunometric assay, using an automated analyzer (Immulyte One, Siemens Healthcare, Milan, Italy). The intra-assay coefficient of variation (CV) of IL-6 was 5.2\% and total imprecision CV $7.4 \%$ at the concentration of $112 \mathrm{ng} / \mathrm{L}$; analytical performance of TNF$\alpha$ were: CV intra-assay $3.5 \%$ and inter-assay CV 5.8\% at the concentration of $34 \mathrm{ng} / \mathrm{L}$. The analytical sensitivity of IL-6 is $2.0 \mathrm{ng} / \mathrm{L}$ and TNF- $\alpha 1.7 \mathrm{ng} / \mathrm{L}$.

\section{High-sensitivity c-reactive protein (CRP) determination in serum}

C-reactive protein was measured using immune-nephelometric method with polistirene particles coated with a monoclonal antibody in a Siemens Vista System, (Siemens Healthcare, Milan, Italy), imprecision intra-assay CV $4.0 \%$, inter-assay CV $4.4 \%$ at the concentration of $3.5 \mathrm{mg} / \mathrm{L}$. Analytical sensitivity $0.6 \mathrm{mg} / \mathrm{L}$.

\section{Melatonin determination in serum}

Melatonin was measured using radio-immunoassay after extraction with precipitating agent, Hoelzel Diagnostika-biotech (Koln, Germany). Imprecision intra-assay CV 9.7\%, inter-assay CV $10.9 \%$ at the concentration of $42 \mathrm{ng} / \mathrm{L}$. Limit of detection $2.3 \mathrm{ng} / \mathrm{L}$.

\section{Ethics}

The study was approved by the Padova University Hospital Ethics Committee and all participants provided written, informed consent. The study was conducted according to the Declaration of Helsinki (Hong Kong Amendment) and Good Clinical Practice (European) guidelines.

\section{Statistical analysis}

The variables distribution was tested for normality using the Shapiro-Wilks' test. Differences between normally distributed variables were examined by Student's t test/one-way ANOVA; subsequent between-group comparisons were performed using the Tukey test. Differences between non normally distributed variables were examined by Mann-Whitney U test/KruskalWallis ANOVA; subsequent between-group comparisons were performed using the Median test for multiple comparisons. Skin temperature over the study period was analysed by repeated measures ANOVA, by group. Correlation analysis was performed by the Pearson $r$ or Spearman rank tests, as appropriate. The receiver operating characteristic (ROC) analysis was used to test the ability of temperature variability methods to distinguish between groups.

\section{Results}

Seven out of the 53 subjects initially recruited were excluded from one/more analyses sets because of lack of adherence to the protocol, loss of one/more sensors, and onset of fever. Thus 46 subjects completed all study parts.

Inpatients with cirrhosis $(n=13)$ : 9 males; $60.7 \pm 10.8$ years; 2 Child A, 5 Child $B, 6$ Child $C$. The causes of hospitalization were fluid retention/hepato-renal syndrome $(n=5)$, hepatic encephalopathy (3), jaundice (2), suspected spontaneous bacterial peritonitis, which was not confirmed on ascitic tap (2), transaminitis (1). The aetiology was as follows: viral $(n=4)$, alcohol (4), mixed (3), and primary sclerosing cholangitis (2).

Outpatients with cirrhosis $(\mathrm{n}=12)$ : 10 males; $63.2 \pm 11.6$ years; 8 Child A, 4 Child B. The aetiology was as follows: viral $(\mathrm{n}=1)$, alcohol (7), mixed (3), and metabolic (1).

Inpatients without cirrhosis group $(n=11): 8$ males; $74.8 \pm 9.1$ years. Reasons for hospitalization were pulmonary oedema $(n=3)$, anaemia (2), chest pain (2), shortness of breath/pneumonia (1), syncope/pulmonary embolism (1), acute pancreatitis (1), acute renal failure (1).

Healthy volunteers group $(\mathrm{n}=10): 3$ males (all 7 females were post-menopausal); $55.9 \pm 6.4$ years. The demographic/clinical features of the populations are presented in Table 1. Inpatients without cirrhosis were significantly older than healthy volunteers and inpatients with cirrhosis. Pugh/MELD average scores were higher in inpatients compared to outpatients with cirrhosis.

\section{Sleep wake profile}


The sleep-wake profile indices of the populations are presented in Table 2. Inpatients with cirrhosis slept significantly worse compared to healthy volunteers. Daytime sleepiness and diurnal preference were comparable across groups, with the majority of subjects exhibiting moderately morning diurnal preference. Over the experimental period, inpatients with cirrhosis presented significantly longer sleep latency compared to inpatients without cirrhosis.

\section{Proximal and distal skin temperature}

A number of patients were forced to begin/end temperature recording after/before the scheduled times because of clinical or logistic issues. Thus, data were available for all from 16:00 hours of study day 1 until 10:00 hours of study day 2, and analyses refer to this period unless otherwise specified.

The time-course of proximal and distal skin temperature plus the DPG are presented in Figure 2. In- and out-patients with cirrhosis had higher proximal temperature compared to the other two groups (Table 3; Figure 2A). In addition, on visual/qualitative analysis of the curves, the time course of proximal temperature in inpatients with cirrhosis had limited variation compared to the other groups (Figure 2A). On formal analysis, both the factors group and time plus their interaction were significant (group: $\mathrm{F}=3.31, \mathrm{p}<0.05$; time: $\mathrm{F}=15.34, \mathrm{p}<0.001$; group*time: $\mathrm{F}=1.84, \mathrm{p}<0.001)$.

On visual/qualitative analysis of the curves, inpatients with and without cirrhosis had higher distal temperature compared to the other two groups (Table 3; Figure 2B). In addition, while some rhythmicity was observed, the time course of their distal temperature was flatter over time (Figure 2B). On formal analysis, the factor time plus the interaction group*time were significant (group: $\mathrm{F}=0.92$, $\mathrm{p}=0.44$; time: $\mathrm{F}=15.06, \mathrm{p}<0.001$; group*time: $\mathrm{F}=2.07, \mathrm{p}<0.001$ ).

On visual/qualitative analysis of the curves, both in- and outpatients with cirrhosis had significantly lower DPG values compared to the other groups, and DPG reached near-zero values significantly later (Figure 2C). On formal analysis, both the factors group and time plus their interaction were significant (group: $\mathrm{F}=3.17, \mathrm{p}<0.05$; time: $\mathrm{F}=7.58, \mathrm{p}<0.001$; group*time: $\mathrm{F}=1.70$, $\mathrm{p}=0.001$ ). Only healthy volunteers exhibited the physiological, obvious change in DPG expected around sleep onset time (Figure 2C). The time course of DPG in inpatients without cirrhosis was closer to that of healthy volunteers, but still flattened (Figure 2C).

For ease of interpretation, temperature results for patients with cirrhosis as a group (as opposed to in- and outpatients) are presented in Supplementary Figure 1.

A significant correlation was observed between minimum proximal temperature value and MELD score $(r=0.54, p<0.01)$. Significant correlations were also observed between temperature and sleep-wake variables when all groups were considered (i.e. minimum proximal temperature vs. sleep onset latency: $r=0.38, p<0.05)$. The relationships held true when analyses were confined to patients with cirrhosis (Table 4).

\section{Temperature variability analysis}

Sample entropy of temperature data was significantly higher in inpatients with cirrhosis group compared to other groups (Figure 3). Inpatients with cirrhosis showed increased entropy compared to all other groups when MSE was used to calculate complexity of temperature signals at different scales (Supplementary Table 1). ROC analysis showed that sample entropy could distinguish between in- and outpatients with cirrhosis with an area under the curve of 0.88 $(\mathrm{p}<0.001)$. MSE demonstrated even better separation [i.e. area under curve (for scaling point 4) $0.94(\mathrm{p}<0.0001)]$. 


\section{Laboratory variables}

On average, inpatients with cirrhosis had higher inflammatory markers compared to both outpatients with cirrhosis and inpatients without cirrhosis (IL6: $56.13 \pm 63.65$ vs. $10.31 \pm 10.15$, and $56.13 \pm 63.65$ vs. $5.52 \pm 3.23 \mathrm{ng} / \mathrm{l}$, respectively; CRP: $43.17 \pm 45.92$ vs. $4.72 \pm 5.81$ and $43.17 \pm 45.92$ vs. $6.53 \pm 8.12 \mathrm{mg} / \mathrm{l}$, respectively). Average morning melatonin levels were also higher in inpatients with cirrhosis but variability was considerable, and the differences not significant $(36.22 \pm 54.88$ vs $28.45 \pm 37.19$ and $36.22 \pm 54.88$ vs $9.11 \pm 6.23 \mathrm{ng} / \mathrm{l})$.

Significant, consistent correlations were observed between inflammatory markers/melatonin levels and temperature (i.e. minimum proximal temperature vs. IL-6: $r=0.46, p<0.01$; minimum proximal temperature vs. CRP: $r=0.42, \mathrm{p}<0.05$; maximum proximal temperature vs. melatonin $\mathrm{r}=0.41 \mathrm{p}<0.05)$. The relationships held true when analyses were confined to patients with cirrhosis (i.e. minimum proximal temperature vs. IL-6: $r=0.49$, $p<0.05$; minimum proximal temperature vs. CRP: $r=0.45, \mathrm{p}<0.05)$.

\section{Discussion}

Diverse theories have been proposed to clarify the thermophysiological mechanism leading to sleep initiation. Over the past decades, the influence of altering core body temperature, body heat production and/or heat loss have been studied as potential processes involved in the circadian regulation of the sleep-wake cycle.26-30 Currently, it is assumed that the redistribution of heat from proximal to peripheral sites, driven by vasodilation of distal skin regions, is a crucial step and it is closely linked to sleep onset latency.3,5,28 Indeed, it has been demonstrated that the DPG is strongly correlated with sleep propensity. 3,5

In the present study the curves of distal and proximal skin temperature recorded in patients with cirrhosis (in- and outpatients) were remarkably different from those observed in inpatients without cirrhosis and healthy volunteers. Considering that environmental conditions and sleep timing were not controlled for, the temperature curves registered in healthy volunteers were substantially comparable to those obtained in other studies.3,5 On the other hand, the circadian rhythm of temperature was abnormal in patients with cirrhosis, particularly inpatients, in whom the temperature curves recorded were markedly blunted. This was more obvious in the proximal temperature curve. The elevated skin temperatures and the absence of circadian variations throughout the day may be explained by the hyperdynamic circulatory syndrome, 31 caused by splanchnic and systemic vasodilation, which is responsible for increased splanchnic blood flow, and also increased skin blood flow.32 Cutaneous vasodilatation is a well-documented phenomenon in patients with liver disease 32 that may explain why patients with cirrhosis had a higher proximal temperature in comparison with healthy volunteers. The reason for systemic vasodilatation in cirrhosis is twofold: 1 . cirrhosis is associated with increased level of circulating endogenous vasodilators; 32 2. vascular responsiveness to adrenergic stimulation is significantly impaired in patients with cirrhosis.33 In addition to blunted response to adrenergic stimulation, vascular beds from cirrhotic subjects have impaired endothelium-dependent vasodilator response to cholinergic stimulation. 34 This indicates that although peripheral vessels in cirrhosis are more dilated than normal individuals, they may not respond well to cholinergic vasodilatatory mechanisms such as sympathetic cholinergic fibers. This is particularly important in cutaneous circulation as the autonomic nerve fibers in the skin almost completely derive from sympathetic cholinergic neurons. 35

The DPG is a good indicator of the time taken to fall asleep so that, when the DPG is near-zero, sleep onset is imminent.3,5 In inpatients with cirrhosis the DPG did never reach zero, whereas in outpatients with cirrhosis a near-zero DPG was recorded almost two hours after the try-to-sleep time they reported. These results suggest a compromised skin thermoregulatory function in patients with cirrhosis. Since the vasodilatation from proximal to distal sites is a crucial physiological step for sleep initiation, the generalized state of vasodilatation which characterizes cirrhosis may hamper heat dissipation. Indeed, such an ineffective heat loss may explain why these patients do not reach a near-zero DPG value and their difficulties in falling asleep. It is also 
interesting to note that the DPG curves obtained in in- and outpatients with cirrhosis were overlapping, and different from both healthy volunteers and inpatients without cirrhosis, suggesting that the aforementioned temperature disturbances are specific to the pathology. Inpatients without cirrhosis were introduced into the study to check for the potential influence of hospitalization on the circadian rhythm of temperature since bed rest and physical inactivity strongly influence heat production/loss.36 Despite the fact that it was not as evident as in healthy volunteers, circadian variations were observed in both distal and proximal temperature curves in inpatients without cirrhosis. Likewise, the DPG was very similar to that obtained in healthy volunteers and tended to zero around sleep onset time, although the curve was somewhat flattened. These findings suggest that bed rest and physical inactivity, together with disease, may influence the circadian rhythm of skin temperature. However, despite the severity of the medical conditions within the inpatients without cirrhosis group, the effect was not sufficient to deeply alter the circadian rhythm, in contrast to what happened in in- and out-patients with cirrhosis. The disturbances in sleep-wake and sleep timing indices observed in the current study in patients with cirrhosis are in line with those previously reported by other authors.6,7,37 Similarly, high morning levels of melatonin have also been reported in this patient population.9,38 Given the importance of thermoregulation in sleep initiation, correlation analyses were conducted and corroborated the existence of an association between sleep disturbances and thermoregulatory alterations observed in these patients, with particularly consistent correlations between sleep timing indices and minimal proximal temperature. Under healthy conditions, body temperature exhibits complex short-term and long-term fluctuations that are due to the nonlinear interplay between sensors and effectors within our thermoregulatory network. Thus, in addition to absolute temperature values, temperature variability analysis gives valuable information about the thermoregulatory system.23,24 In the present study, we estimated the complexity of temperature fluctuations by calculating sample entropy as well as MSE of the temperature series. Our results demonstrated that among our experimental groups, healthy volunteers, outpatients with cirrhosis and inpatients without cirrhosis had similar sample entropy and MSE in their temperature fluctuations. The only group that had significantly higher entropy was inpatients with cirrhosis. According to Pincus, 39 tighter coupling of signals within a complex interconnected system increases the entropy of the system. In other words, increased entropy may indicate that different components of a system are engaged and coupled together. This increased entropy in the temperature series might be an indicator of an active process that has engaged the thermoregulatory system. In this respect, inpatients with cirrhosis also had higher serum levels of CRP and cytokines, which suggests that systemic inflammation may be a factor associated with their admission into a tertiary referral liver unit. ROC analysis showed that both MSE and sample entropy are very sensitive and specific indices for distinguishing between in- and outpatients with cirrhosis, with an area under curve higher than $88 \%$. This goes along with the idea that nonlinear analysis of temperature time series may provide a quantitative measure for assessment of the thermoregulatory circuit in clinical practice. 23 The observation of abnormalities in both absolute temperature values and their dynamics has significant clinical ramifications, especially on the concept of fever.

Our study has a number of limitations. Firstly, it is an observational study, and finding a significant association between impaired skin temperature gradient and sleep onset does not necessarily indicate causality. The relationship between DPG and sleep abnormalities is interesting but might have been influenced by unknown confounders. Secondly, because of the known effect of beta-blockers on melatonin secretion, patients on beta-blockers were excluded, thus making the population in this study only partially representative of patient with cirrhosis as a group.

In conclusion, considerable alterations of distal/proximal skin temperature and their time-course were observed in patients with cirrhosis. Such alterations seem to parallel the severity of the disease and the associated sleep-wake disturbances. These findings may have implications for the definition of fever and the management of insomnia in these patients. 


\section{Acknowledgements}

The authors are grateful to Professor Eus van Someren (Netherlands Institute for Neuroscience, Amsterdam) for technical support and generous advice on outliers removal.

\section{References}

1. Borbély AA. A two process model of sleep regulation. Hum Neurobiol 1982;1:195-204.

2. Borbély AA. From slow waves to sleep homeostasis: new perspectives. Arch Ital Biol 2001;139:53-61.

3. Kräuchi K, Cajochen C, Werth E, Wirz-Justice A. Functional link between distal vasodilatation and sleep-onset latency?. Am J Physiol Regulatory Integrative Comp Physiol 2000;278:R741R748.

4. Kräuchi K, Deboer T. The interrelationship between sleep regulation and thermoregulation. Front Biosci (Landmark Ed) 2010;15:604-625.

5. Kräuchi K, Cajochen C, Werth E, Wirz-Justice A. Warm feet promote the rapid onset of sleep. Nature 1999;401:36-37.

6. Córdoba J, Cabrera J, Lataif L, Penev P, Zee P, Blei AT. High prevalence of sleep disturbance in cirrhosis. Hepatology 1998;27:339-345.

7. Mostacci B, Ferlisi M, Baldi Antognini A, et al. Sleep disturbance and daytime sleepiness in patients with cirrhosis: a case control study. Neurol Sci 2008;29:237-240.

8. De Rui M, Schiff S, Aprile D, et al. Excessive daytime sleepiness and hepatic encephalopathy: it is worth asking. Metab Brain Dis 2013;28:245-248.

9. Montagnese S, Middleton B, Mani AR, Skene DJ, Morgan MY. On the origin and the consequences of circadian abnormalities in patients with cirrhosis. Am J Gastroenterol 2010;105:1773-1781.

10. Boy C, Meyer PT, Kircheis G et al. Cerebral A1 adenosine receptors (A1AR) in liver cirrhosis. Eur J Nucl Med Mol Imaging 2008;35:589-597.

11. Bersagliere A, Raduazzo ID, Nardi M, et al. Induced hyperammonemia may compromise the ability to generate restful sleep in patients with cirrhosis. Hepatology 2012;55:869-878.

12. Montagnese S, De Pittà C, De Rui M, et al. Sleep-wake abnormalities in patients with cirrhosis. Hepatology 2014;59:705-712.

13. Pugh RN, Murray-Lyon IM, Dawson JL, Pietroni MC, Williams R. Transection of the oesophagus for bleeding oesophageal varices. Br J Surg 1973;60:646-649.

14. Kamath PS, Wiesner RH, Malinchoc M, et al. A model to predict survival in patients with end-stage liver disease. Hepatology 2001;33:464-470.

15. Buysse DJ, Reynolds CF III, Monk TH, Berman SR, Kupfer DJ. The Pittsburgh sleep quality index: a new instrument for psychiatric practice and research. Psychiatry Res 1989;28:193-213.

16. Curcio G, Tempesta D, Scarlata S, et al. Validity of the Italian version of the Pittsburgh sleep quality index (PSQI). Neurol Sci 2013;34:511-519.

17. Vignatelli L, Plazzi G, Barbato A, et al. Italian version of the Epworth sleepiness scale:

External validity. Neurol Sci 2003;23:295-300.

18. Hörne JA, Östberg OA. Self-assessment questionnaire to determine morningness-eveningness in human circadian rhythms. Int J Chronobiol 1976;4:97-110.

19. Carney CE, Buysse DJ, Ancoli-Israel S, et al. The consensus sleep diary: standardizing prospective sleep self-monitoring. Sleep 2012;35:287-302.

20. van Marken Lichtenbelt WD, Daanen HA, Wouters L, et al. Evaluation of wireless determination of skin temperature using iButtons. Physiol Behav 2006;88:489-497.

21. Rutkove SB, Nie R, Mitsa T, Nardin RA. A methodology for the real-time measurement of distal extremity temperature. Physiol Meas 2007;28:1421-1428.

22. Kräuchi K, Cajochen C, Möri D, Graw P, Wirz-Justice A. Early evening melatonin and S20098 advance circadian phase and nocturnal regulation of core body temperature. Am J Physiol 1997;272:R1178-R1188. 
23. Varela M, Churruca J, Gonzalez A, Martin A, Ode J, Galdos P. Temperature curve complexity predicts survival in critically ill patients. Am J Respir Crit Care Med 2006; 74:290298.

24. Papaioannou VE, Chouvarda IG, Maglaveras NK, Pneumatikos IA. Temperature variability analysis using wavelets and multiscale entropy in patients with systemic inflammatory response syndrome, sepsis, and septic shock. Crit Care 2012;16:R51.

25. Costa M, Goldberger AL, Peng CK. Multiscale entropy analysis of complex physiologic time series. Phys Rev Lett 2002;89:068102.

26. Van Someren EJW. Sleep propensity is modulated by circadian and behavior-induced changes in cutaneous temperature. J Therm Biol 2004;29:437-444.

27. Kräuchi K. The thermophysiological cascade leading to sleep initiation in relation to phase of entrainment. Sleep Med Rev 2007;11:439-451.

28. Raymann RJ, Swaab DF, Van Someren EJ. Cutaneous warming promotes sleep onset. Am J Physiol Regul Integr Comp Physiol. 2005;288:R1589-97.

29. Raymann RJ, Swaab DF, Van Someren EJ. Skin deep: enhanced sleep depth by cutaneous temperature manipulation. Brain. 2008;131:500-13.

30. Romeijn N, Raymann RJ, Møst E, Te Lindert B, Van Der Meijden WP, Fronczek R, GomezHerrero G, Van Someren EJ. Sleep, vigilance, and thermosensitivity. Pflugers Arch.

2012;463:169-76.

31. Bolognesi M, Di Pascoli M, Verardo A, Gatta A. Splanchnic vasodilatation and hyperdynamic circulatory syndrome in cirrhosis. World J Gastroenterol 2014;20:2555-2563. 32. Sacerdoti D, Mania D, Jiang H, Pesce P, Gaiani S, Gatta A, Bolognesi M. Increased EETs participate in peripheral endothelial dysfunction of cirrhosis. Prostaglandins Other Lipid Mediat 2012; 98:129-132.

33. Atucha NM, Shah V, García-Cardeña G, Sessa WE, Groszmann RJ. Role of endothelium in the abnormal response of mesenteric vessels in rats with portal hypertension and liver cirrhosis. Gastroenterology 1996;111:1627-1632.

34. Ostadhadi S, Rezayat SM, Ejtemaei-Mehr S, et al. Mesenteric artery responsiveness to acetylcholine and phenylephrine in cirrhotic rats challenged with endotoxin: the role of TLR4. Can J Physiol Pharmacol 2015;93:475-483.

35. Roosterman D, Goerge T, Schneider SW, Bunnett NW, Steinhoff M. Neuronal control of skin function: the skin as a neuroimmunoendocrine organ. Physiol Rev 2006;86:1309-1379.

36. Kräuchi K, Wirz-Justice A. Circadian rhythm of heat production, heart rate, and skin and core temperature under unmasking conditions in men. Am J Physiol 1994;267:R819-R829.

37. Montagnese S, Middleton B, Skene DJ, Morgan MY. Sleep-wake patterns in patients with cirrhosis: all you need to know on a single sheet. J Hepatol 2009;51:690-695.

38. Steindl PE, Finn B, Bendok B, Rothke S, Zee PC, Blei AT. Disruption of the diurnal rhythm of plasma melatonin in cirrhosis. Ann Intern Med 1995;123:274-277.

39. Pincus SM. Greater signal regularity may indicate increased system isolation. Math Biosci 1994; 122:161-181.

\section{Figure legends}

Figure 1. One experimental session was conducted from 08:00 to 12:00 midday of the following day. Blood samples were collected at the beginning of the experimental session, together with a baseline sleep-wake profile, to include the Pittsburgh Sleep Quality Index (PSQI), the Epworth Sleepiness Scale (ESS) and the Horne-Östberg (HÖ) questionnaire of diurnal preference. Subjects filled a detailed wake diary before going to bed, and a sleep diary on the following morning. Skin temperature was recorded for 24 hours, from 12:00 midday to 12:00 midday of the following day. Figure 2. Proximal skin temperature (mean $\pm 95 \%$ Confidence Interval; panel A), distal skin temperature (panel B) and distal-proximal gradient (DPG; panel C) in healthy volunteers (green 
circles), outpatients with cirrhosis (blue circles), inpatients with cirrhosis (red circles) and inpatients without cirrhosis (orange circles).

On repeated measures ANOVA, by group:

A: group: $F=3.31, p<0.05$; time: $F=15.34, p<0.001$; group*time: $F=1.84, p<0.001$

$B$ : group: $F=0.92, p=0.44$; time: $F=15.06, p<0.001$; group $*$ time: $F=2.07, p<0.001$

$C$ : group: $F=3.17, p<0.05$; time: $F=7.58, p<0.001$; group $*$ time: $F=1.70, p=0.001$

Figure 3. Sample entropy of temperature time-series (mean \pm SEM) in healthy volunteers, outpatients with cirrhosis, inpatients with cirrhosis and hospitalized patients without cirrhosis. $s p<0.001$ in comparison with healthy volunteers and outpatients with cirrhosis; $\# p<0.05$ in comparison with inpatients without cirrhosis.

Supplementary Figure 1. Proximal skin temperature (mean $\pm 95 \%$ Confidence Interval; panel A), distal skin temperature (panel B) and distal-proximal gradient (DPG; panel C) in healthy volunteers (green circles), patients with cirrhosis (pink circles), and inpatients without cirrhosis (orange circles). 
Figure 1

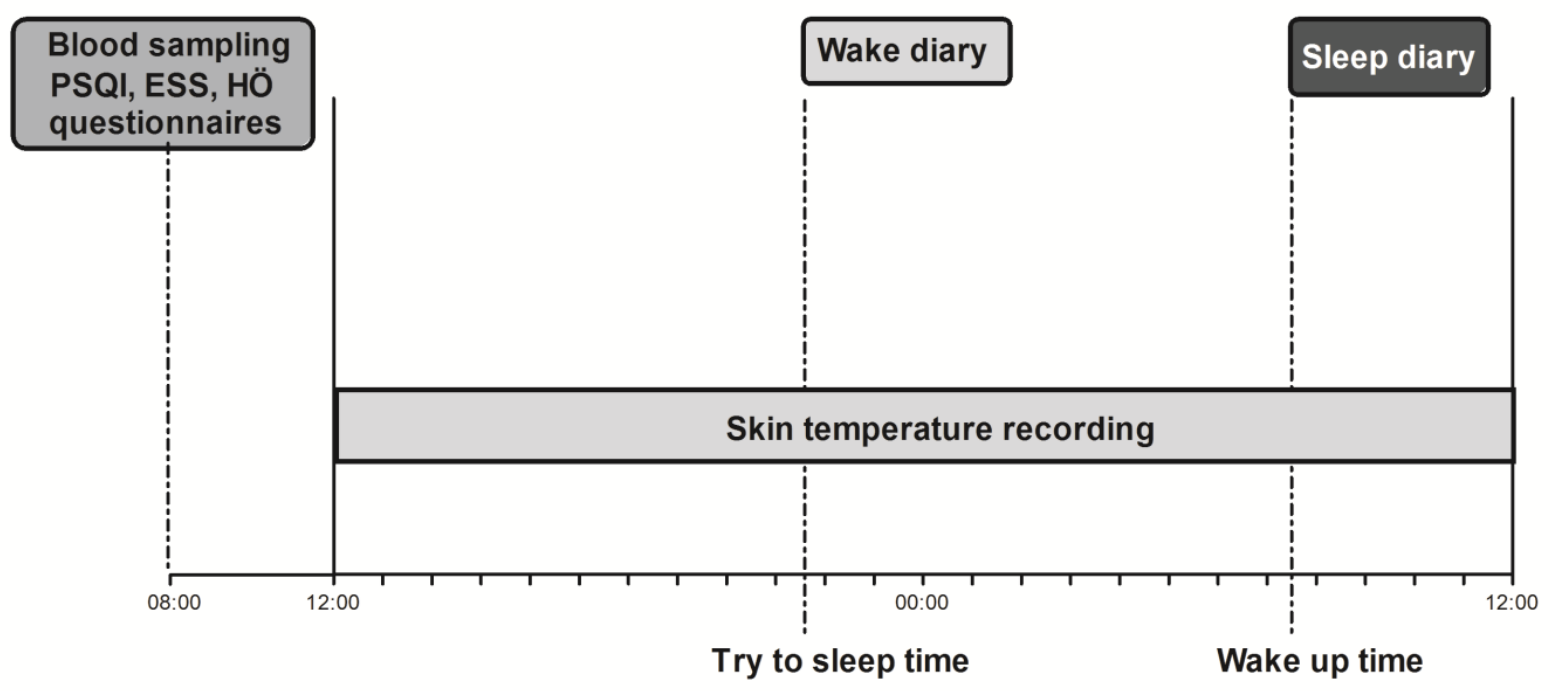

Figure 2A

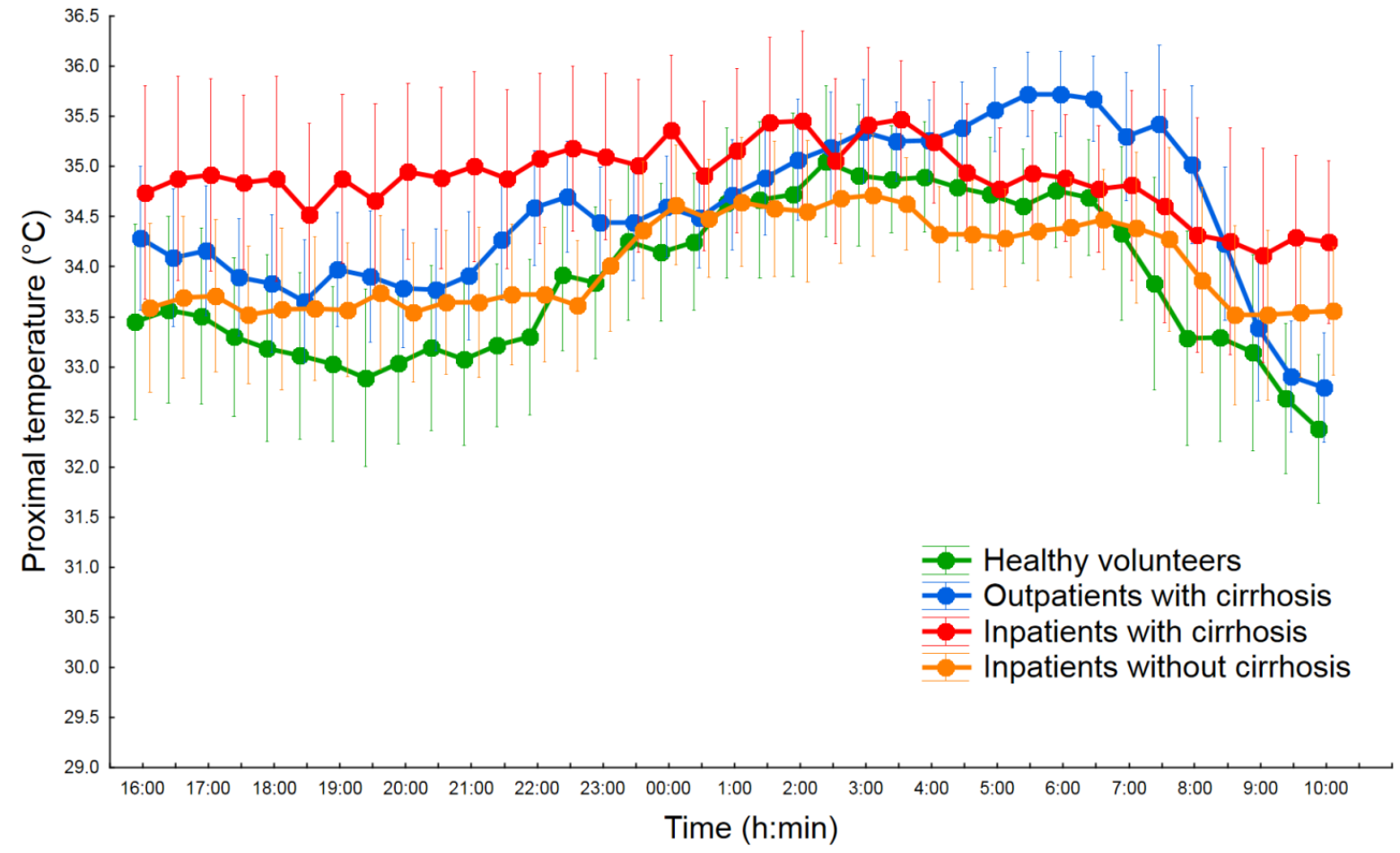


Figure 2B

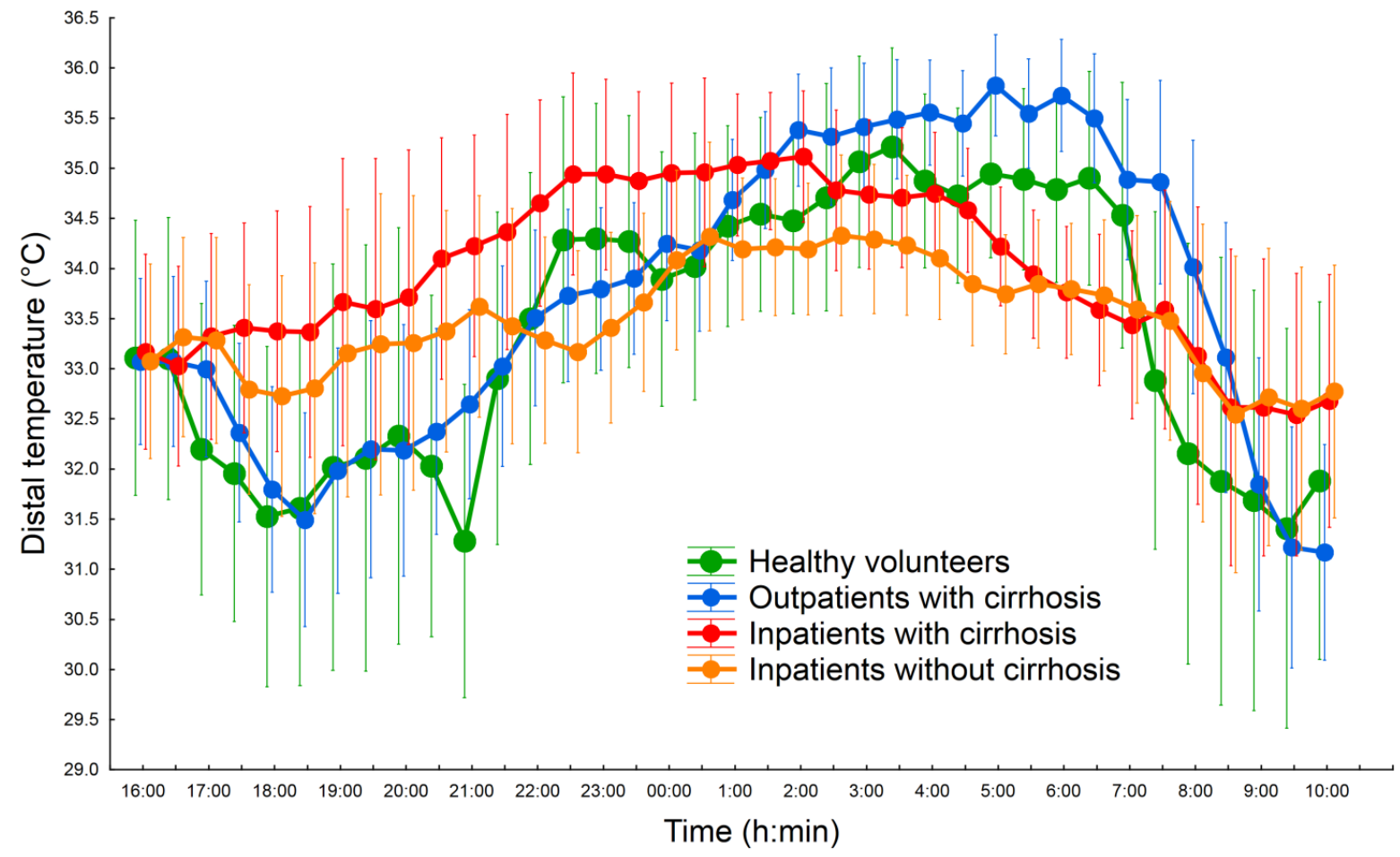

Figure 2C

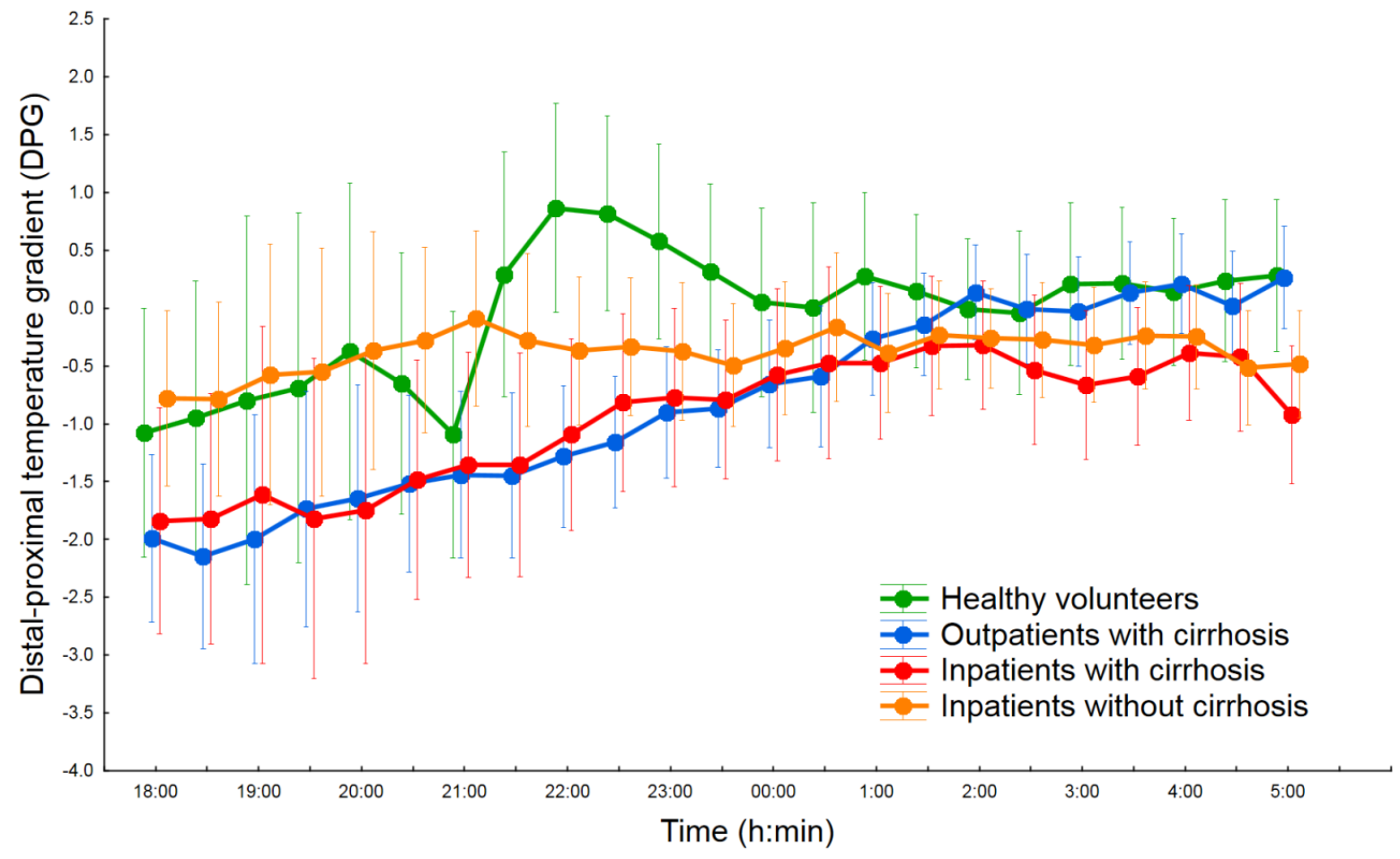


Figure 3

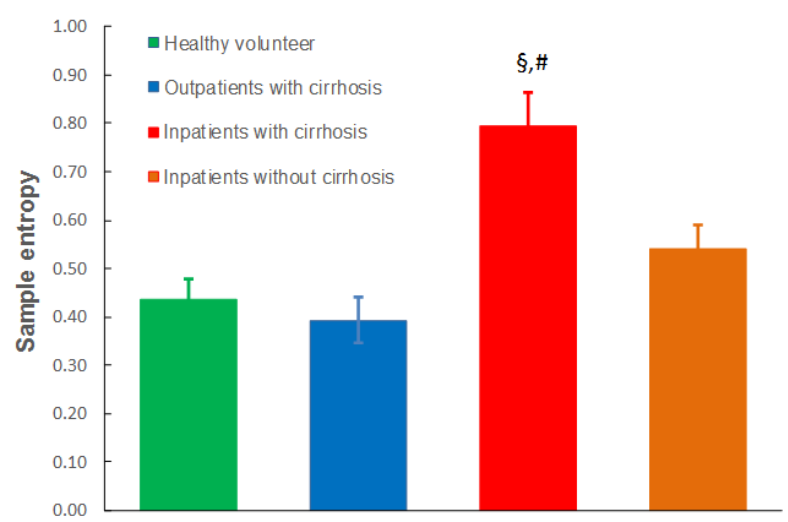

